\title{
Deletion of vanilloid receptor 1-expressing primary afferent neurons for pain control
}

\author{
Laszlo Karai, ${ }^{1}$ Dorothy C. Brown, ${ }^{2}$ Andrew J. Mannes, ${ }^{1}$ Stephen T. Connelly, ${ }^{1}$ Jacob Brown, ${ }^{1}$ \\ Michael Gandal, ${ }^{1}$ Ofer M. Wellisch, ${ }^{1}$ John K. Neubert, ${ }^{1}$ Zoltan Olah, ${ }^{1}$ and Michael J. Iadarola ${ }^{1}$
}

\author{
${ }^{1}$ National Institute of Dental and Craniofacial Research, Bethesda, Maryland, USA. ${ }^{2}$ School of Veterinary Medicine,
} University of Pennsylvania, Philadelphia, Pennsylvania, USA

\begin{abstract}
Control of cancer, neuropathic, and postoperative pain is frequently inadequate or compromised by debilitating side effects. Inhibition or removal of certain nociceptive neurons, while retaining all other sensory modalities and motor function, would represent a new therapeutic approach to control severe pain. The enriched expression of transient receptor potential cation channel, subfamily V, member 1 (TRPV1; also known as the vanilloid receptor, VR1) in nociceptive neurons of the dorsal root and trigeminal ganglia allowed us to test this concept. Administration of the potent TRPV1 agonist resiniferatoxin (RTX) to neuronal perikarya induces calcium cytotoxicity by opening the TRPV1 ion channel and selectively ablates nociceptive neurons. This treatment blocks experimental inflammatory hyperalgesia and neurogenic inflammation in rats and naturally occurring cancer and debilitating arthritic pain in dogs. Sensations of touch, proprioception, and high-threshold mechanosensitive nociception, as well as locomotor function, remained intact in both species. In separate experiments directed at postoperative pain control, subcutaneous administration of RTX transiently disrupted nociceptive nerve endings, yielding reversible analgesia. In human dorsal root ganglion cultures, RTX induced a prolonged increase in intracellular calcium in vanilloid-sensitive neurons, while leaving other, adjacent neurons unaffected. The results suggest that nociceptive neuronal or nerve terminal deletion will be effective and broadly applicable as strategies for pain management.
\end{abstract}

\section{Introduction}

In patient populations ranging from pediatric to the elderly, control of severe pain with currently available analgesics is frequently less than optimal (1-3). Opioids remain the mainstay of treatment for moderate to severe pain, but they cannot universally provide relief. Some patients with cancer have pain that is refractory to conventional medical therapies including opioid analgesia, and many of them experience aversive, dose-limiting side effects. Despite more than 100 years of searching for new pharmacological modalities to treat severe pain (4-6), only very few have emerged, and the current alternatives to opioids are nonselective chemical or surgical neuroablative interventions and palliative sedation (7-9). Both options are unsatisfactory, however. With neuroablative interventions, neurons responsible for motor, bladder, and bowel function may be indiscriminately destroyed, while with palliative sedation there is loss of consciousness; in both cases, quality of life is potentially compromised (10). Implantable pumps delivering continuous intrathecal administration of drugs may offer relief, but pump placement is expensive, requires a surgical procedure, has its own set of complications, and may not be appropriate or completely effective for advanced stages of cancer (11).

In the present article, we explore selective nociceptive neuronal deletion as an approach to control nociceptive processes in experimental models and naturally occurring clinical conditions. This

Nonstandard abbreviations used: calcitonin gene-related peptide (CGRP); capsaicin (CAP); dorsal root ganglion (DRG); free intracellular calcium concentration $\left(\left[\mathrm{Ca}^{2+}\right]_{\mathrm{i}}\right)$; immunoreactive (IR); resiniferatoxin (RTX); transient receptor potential cation channel, subfamily V, member 1 (TRPV1); trigeminal ganglion (TG); visual analog scale (VAS).

Conflict of interest: The authors have declared that no conflict of interest exists.

Citation for this article: J. Clin. Invest. 113:1344-1352 (2004).

doi:10.1172/JCI200420449 approach takes advantage of specific gene expression patterns in nociceptive, mainly small unmyelinated C-fiber neurons localized in sensory ganglia. These neurons transduce sensations generated by noxious heat and endogenous inflammatory algesic substances and express high levels of the transient receptor potential cation channel, subfamily V, member 1 (TRPV1; also known as the vanilloid receptor, VR1), a sodium/calcium ion channel, as compared with other sensory neurons in the dorsal root (DRG) or trigeminal ganglia (TG) (12-14). Direct live-cell imaging of primary DRG cultures and ectopically TRPV1-expressing cells has demonstrated that resiniferatoxin (RTX), a potent TRPV1 agonist (15), causes a large, prolonged increase in the free intracellular calcium concentration $\left(\left[\mathrm{Ca}^{2+}\right]_{i}\right)$. The resulting calcium cytotoxicity rapidly compromises and then deletes only the TRPV1-expressing neurons or cells (16). These data provided a mechanistic rationale for therapeutic intervention in which application of RTX in the vicinity of DRG or TG neuronal cell bodies in vivo would delete TRPV $1^{+}$neurons, thereby eliminating sensitivity to nociceptive input associated mainly with inflammation, hyperalgesia, and cancer-related pain. Other sensory modalities mediated by neurons not expressing TRPV1 would remain unaffected (e.g., proprioception and high-threshold mechanosensation). We reasoned further that the same mechanism applied to nociceptive nerve terminals in the periphery would reversibly inhibit pain transduction but spare neuronal perikarya in the DRG, which are remote from the site of RTX application. Thus, three strategies for pain modulation based on differential application of RTX were investigated: single intraganglionic, multiganglionic (intrathecal), and peripheral nerve terminal. We employed robust, well-characterized nociceptive experimental models in rats, as well as a canine veterinary model presenting with naturally occurring conditions (cancer, osteoarthritis) that have direct relevance 

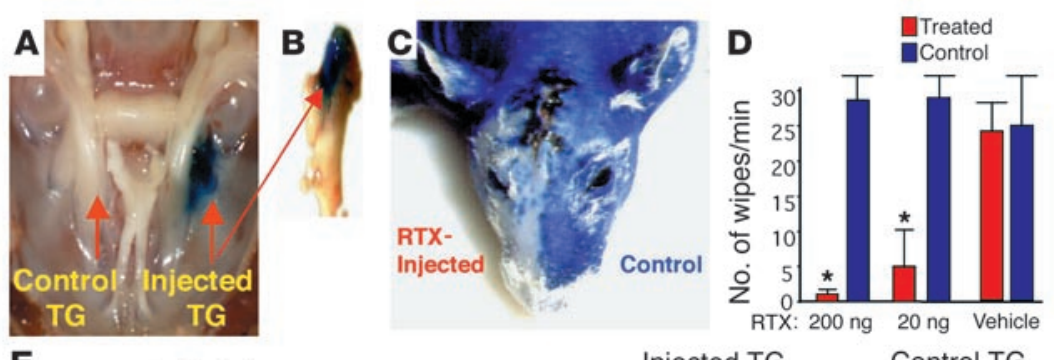

E
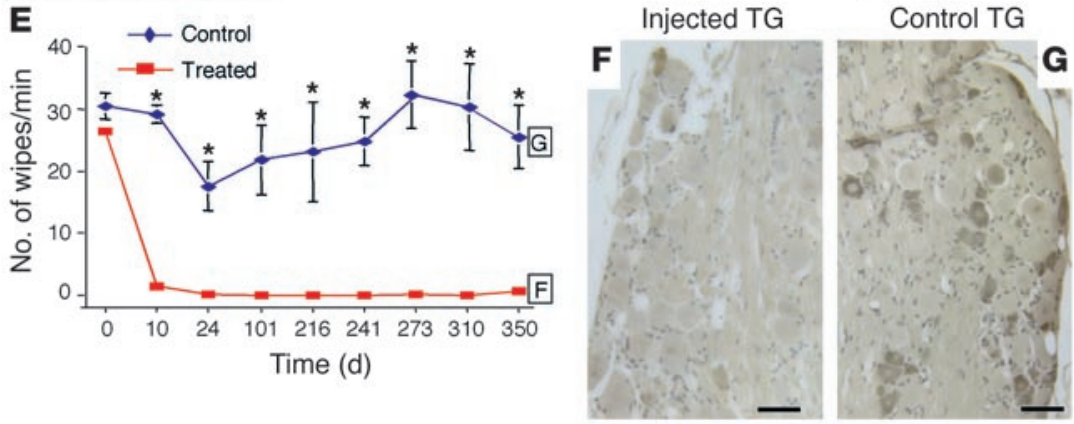

Figure 1

Single intraganglionic treatment. RTX was microinjected unilaterally into the TG using a transcranial stereotaxic approach. (A) Coomassie blue dye depicts trigeminal injection site at the base of the skull and (B) excised, stained TG. (C) RTX-induced blockade of neurogenic inflammation was evaluated by extravasation of Evans blue-stained plasma proteins. Blue areas of skin identify regions with intact $\mathrm{C}$-fiber innervation. Extravasation was blocked on the RTX-injected side, which remains white. (D) Dose-related blockade of nociceptive afferent transmission from the cornea after unilateral intraganglionic RTX administration. CAP eye-wipe response was assessed 1-3 days after injection. *Pairwise $t$ test; $P<0.01 ; n=30,6$, and 18 for administration of $200 \mathrm{ng}, 20 \mathrm{ng}$, and vehicle, respectively. (E) Block of CAP-induced eye-wipe response is evident as up to about 1 year in unilaterally intraganglionically treated rats, consistent with the permanent deletion of TRPV1-IR neurons, as shown in $\mathbf{F}$ and $\mathbf{G}$ ( ${ }^{*}$ repeated pairwise $t$ test; $P<0.01 ; n=7$ ). On the RTX-treated side $(\mathbf{F})$, there is a reduction $(\sim 80 \%$, similar to animals examined within 1 to 3 days after RTX treatment; see Figure 2) of TRPV1-IR neurons compared with the contralateral nontreated side (G). Bars: $100 \mu \mathrm{m}$.

to humans. To predict efficacy and establish whether the same selectivity would occur in humans, we exposed primary cultures of human DRG neurons to RTX. Live-cell imaging disclosed that only a subpopulation of DRG neurons exhibited activation with RTX; this is consistent with previous results in cultured rat DRG neurons. The procedures outlined should provide analgesia to a variety of disorders that are characterized by severe pain and that may include trigeminal neuralgia, arthritis, peripheral neuromas, and advanced metastatic disease.

\section{Results}

Single intraganglionic injection in rat experimental models. Unilateral intraganglionic microinjection of RTX into the TG (Figure 1, A and $\mathrm{B}$ ) attenuated or eliminated both the afferent (nociceptive) and efferent (neurogenic inflammation) functions supported by C-fiber neurons (Figure 1, C-E). Intraganglionic RTX attenuated plasma extravasation; this is an indicator of neurogenic inflammation and efferent function mediated by C fibers (17, 18). Intravenous Evans Blue dye, which binds to plasma proteins, delineated the areas with intact innervation (Figure 1C), staining them deep blue. The skin remained largely white on the RTXmicroinjected side, where trigeminal neurons expressing TRPV1 were deleted. Analgesic actions were observable as soon as 24 hours after injection using a sensitive test for C-fiber function, the eye-wipe response to corneal application of capsaicin (CAP). An intraganglionic dose of 20 ng of RTX nearly eliminated the wiping behavior, and complete suppression was obtained with $200 \mathrm{ng}$ (Figure 1D). The antinociceptive effects from a single RTX injection were maintained for at least 1 year (Figure 1E). The longduration behavioral effect and loss of TRPV1immunoreactive (TRPV1-IR) neurons (Figure 1, F and G) suggest that RTX analgesia is the result of permanent cell removal and not the result of prolonged receptor desensitization; the latter phenomenon is observed when CAP is applied to neurons in acute in vitro electrophysiological experiments (19-21). The loss of CAP chemosensitivity did not affect the mechanosensitive aspects of the corneal reflex to the liquid droplet itself. Furthermore, there were no observable alterations in grooming that might indicate the presence of a sensory dysesthesia.

That intraganglionic RTX administration produced extensive deletion of TRPV1-expressing neurons could be seen by immunohistochemistry as early as 1-3 days after RTX treatment (Figure 2, A-D). To establish that another class of sensory ganglion neurons remains intact, we stained for a neurofilament protein (N52) expressed in neurons with large-diameter myelinated axons (22). Both single- and double-stained (N52 and TRPV1) sections were examined. Injected TGs contain many $\mathrm{N} 52^{+}$neurons $(684 \pm 32$, brown in Figure 2, $\mathrm{C}$ and $\mathrm{D})$ but very few $\mathrm{TRPV} 1^{+}$neurons $(123 \pm 36$, purple), while contralateral TGs show both N52 $(709 \pm 23)$ and TRPV1 ${ }^{+}(604 \pm 68)$ neurons (Figure 2 , E and F). The loss of TRPV1 ${ }^{+}$neurons was significant $(P<0.01)$. The loss was also evident using RT-PCR; however, a small amount of RNA TRPV1 $_{\text {was detectable in the }}$ RTX-injected ganglia (Figure 2G), consistent with cell counts showing substantial loss of TRPV1-IR neurons and retention of the N52 neurons not expressing TRPV1 (Figure 2, C and D).

Multiganglionic administration of RTX in rats. Severe pain secondary to advanced metastatic disease is generally more diffuse and not localized to one or two dermatomes. In these cases, multiple ganglia can be treated bilaterally via the cerebrospinal fluid. To test this, RTX was administered by lumbar puncture to target ganglia that innervate the tail and lower limbs. Treatment effectiveness and specificity were assessed by measuring (a) selective attenuation of noxious thermal and inflammatory responses, (b) the degree of spread of the drug by behavioral comparison to the forelimbs, corresponding to the spatial extent of the cell deletion, and (c) retention of locomotor activity and mechanosensation. RTX administered intrathecally $(10,50 \mathrm{ng})$ produced minor increases in withdrawal latency to a radiant noxious thermal stimulus (23, 24), whereas at doses of 100 and $200 \mathrm{ng}$, many animals reached the 14-second cutoff for hindpaw and tail stimulation without affecting forepaw latencies (Figure 3A). Intrathecal RTX also blocked carrageenan-induced thermal hyperalgesia, consistent with a substantial role of TRPV1 ${ }^{+}$neurons in experimental inflammatory conditions (Figure $3 \mathrm{~B}$ ), as shown by targeted disruption of the TRPV1 gene $(25,26)$. Behavioral effects were reflected at the cellu- 
RTX-treated TG
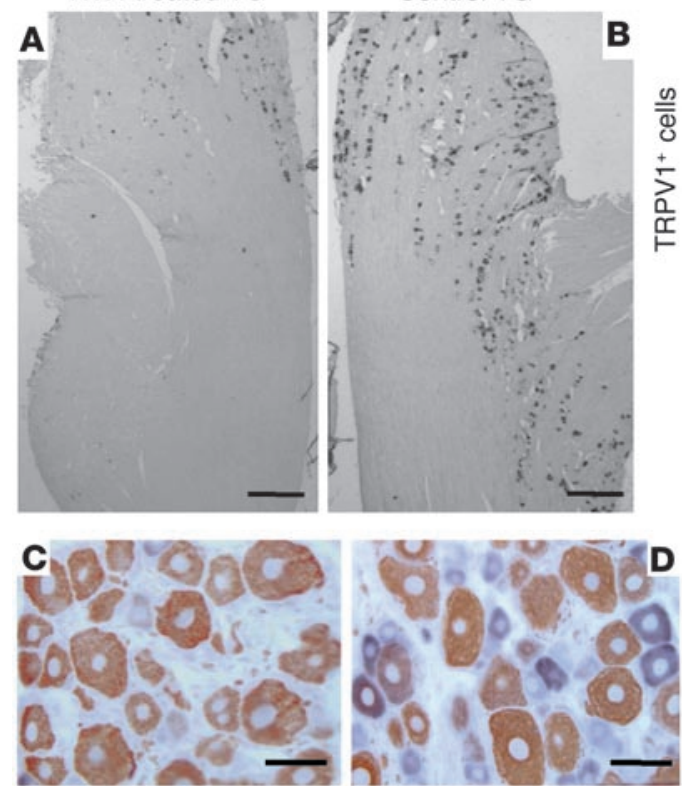

Control TG

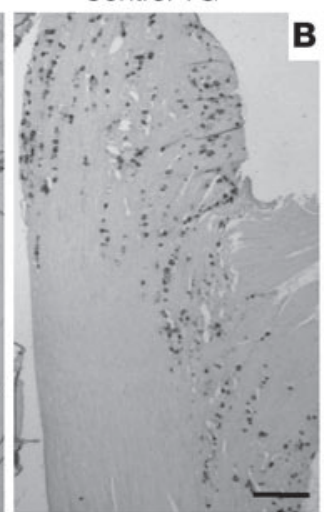

B
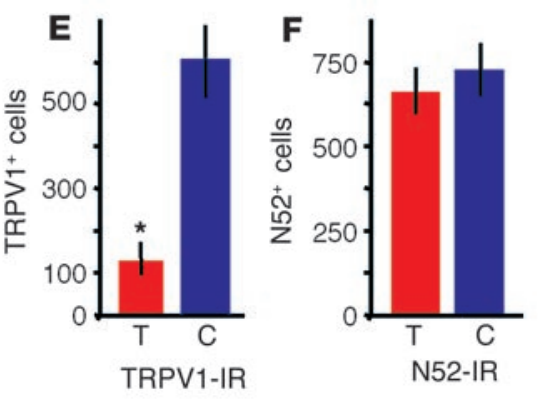

G

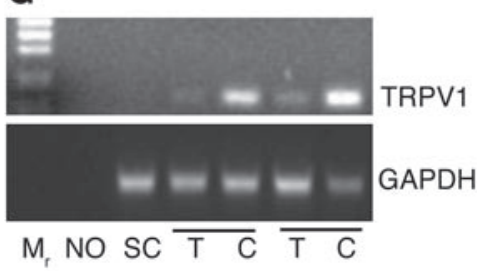

Figure 2

Selective loss of TRPV1-IR sensory ganglion neurons after RTX (200 ng) microinjection. (A) Immunostaining for TRPV1 shows extensive loss of IR neuronal perikarya after RTX injection compared with contralateral noninjected TG (B). (C-F) Double labeling shows that large myelinated $\mathrm{N} 52^{+}$sensory neurons (brown) are retained on the RTX-injected side, whereas TRPV1+ neurons (purple) are deleted (C). On the contralateral, noninjected side, both N52 and TRPV1 neurons are intact (D). Quantification shows no significant difference in the number of N52-IR perikarya after RTX, whereas an $80 \%$ reduction in TRPV $1^{+}$neurons occurs (E and $\mathbf{F}$ ). Bars in graph represent the average neuron counts in three sections of TG from three to five different rats assessed between 1 and 3 days after injection $\left({ }^{*} P<0.01\right)$. (G) RT-PCR shows reduction of $\mathrm{mRNA}_{\mathrm{TRPV}} 1$ in two different rats. $\mathrm{M}_{\mathrm{r}}$, markers; NO, no primer; $\mathrm{SC}$, spinal cord; T, RTX treated; $\mathrm{C}$, contralateral TG. Bars: 0.5 $\mathrm{mm}(\mathbf{A}$ and $\mathbf{B})$ and $50 \mu \mathrm{m}(\mathbf{C}$ and $\mathbf{D})$.

lar level by decreases in TRPV1 and calcitonin gene-related peptide (CGRP) immunoreactivities in the lumbar ganglia and dorsal horn, respectively, consistent with deletion of TRPV1-expressing neurons (Figure 3, D-G). In contrast, TRPV1 ${ }^{+}$neurons in cervical ganglia, which are remote from the level of lumbar RTX application, were unaffected (Figure 3C), corresponding to retention of normal forepaw-withdrawal latencies (Figure 3A). Thus, by targeting one or many ganglia, using the intraganglionic or intrathecal routes, respectively, the spatial extent of the therapeutic action may be adjusted to match varied clinical presentations.

Retention of other somatosensory functions and locomotion. Importantly, the response to mechanical stimuli remained intact. Orientation to and withdrawal from a pinch with toothed forceps on the tail, forepaws, and hindpaws were present in all RTX-treated animals, and responses were qualitatively similar to controls. Quantitative assessment of mechanosensation using von Frey hairs also showed no alteration in RTX-treated ( 3 and 10 days and 1 year) animals in comparison to control animals. No significant difference was observed in the threshold for light touch or for paw withdrawal between untreated controls $(10.9 \pm 1.8 \mathrm{~g}$ for light touch, $20.3 \pm 5.7$ $\mathrm{g}$ for paw withdrawal), treated animals after 3 days $(11 \pm 1.8 \mathrm{~g}$, $46 \pm 30.6 \mathrm{~g})$, treated animals after 10 days $(10.1 \pm 1.1 \mathrm{~g}, 22 \pm 4.6 \mathrm{~g})$ and treated animals after 1 year $(11.7 \pm 0.0 \mathrm{~g}, 33.7 \pm 16 \mathrm{~g})$. There were no indications of mechanoallodynia such as prolonged withdrawal to touch with the von Frey hairs, or mechanohyperalgesia as assessed by withdrawal from a pinprick (all animals had a withdrawal duration of 0.5 second or less) (27).

No alteration occurred in locomotor function as evaluated with an accelerating Rota-Rod, indicating the presence of intact motor axons and sensory proprioceptive neurons in ganglia exposed to RTX (Figure 3, D and H). Normal locomotion was also evident in the canine subjects (see later). As with the trigeminal microinjections, no indications of sensory neglect or denervationinduced hyperesthesia syndromes (e.g., autotomy behavior) were observed (28, 29). These features reinforce the clinical applicability of RTX, since the pathological, inflammation-associated aspects of pain are eliminated, while mechanonociception, motor function, and other sensory modalities remain intact.

Peripheral RTX administration. The same concept of ligand-activated calcium cytotoxicity was applied to the peripheral nerve terminals to obtain a transient reduction of nociceptive transmission. Injection of 100 ng RTX subcutaneously into the hindpaw significantly attenuated thermal nociception for approximately 20 days. The analgesic activity was confined to the injected hindpaw, and no alteration in latency was detected for the contralateral paw or the forepaws (Figure 3I). Since the injection was remote from the cell body, it was predicted that the effect would be transient, and that TRPV1 ${ }^{+}$cells would remain intact; both of these predicted results were observed (Figure 3 , I-K). Counts of TRPV1-IR neurons from eight sections of L5 ganglia showed $119 \pm 23$ neurons on the injected side and $113 \pm 10$ on the contralateral side. Thus, the dose and anatomical site of RTX application critically determine whether the analgesic action will be reversible, as would be desired for postoperative pain, or irreversible (e.g., terminal cancer pain) $(30,31)$.

Veterinary clinical application. The canine model was established to assess the efficacy of primary afferent neuronal deletion in a higher order mammal with clinical problems more closely approximating the human situation. On the basis of attenuation of thermal response determined in an initial cohort, a single intrathecal dose of RTX $(1 \mu \mathrm{g} / \mathrm{kg})$ was administered under general anesthesia via cisternal puncture. Visual analog scale (VAS) ratings of nociceptive status before the injection averaged $62 \pm 7.6$ (mean \pm SEM); these ratings sharply decreased $(11 \pm 3.0)$ at the 2 -week follow-up and were maintained at 6 and 10 weeks $(9.6 \pm 5.3$ and $7.5 \pm 4.2$, respectively) (Figure 4A). The animals initially presented with limb guarding as they walked, but this improved over time and daily activity increased, as was evident from video recordings. In fact, the entire demeanor of the dogs appeared improved following intrathecal RTX treatment. The efficacy of RTX action was further demonstrated by (a) the discontinuation or greatly reduced use of supplementary analgesics (opioids and NSAIDs in all eight dogs) 

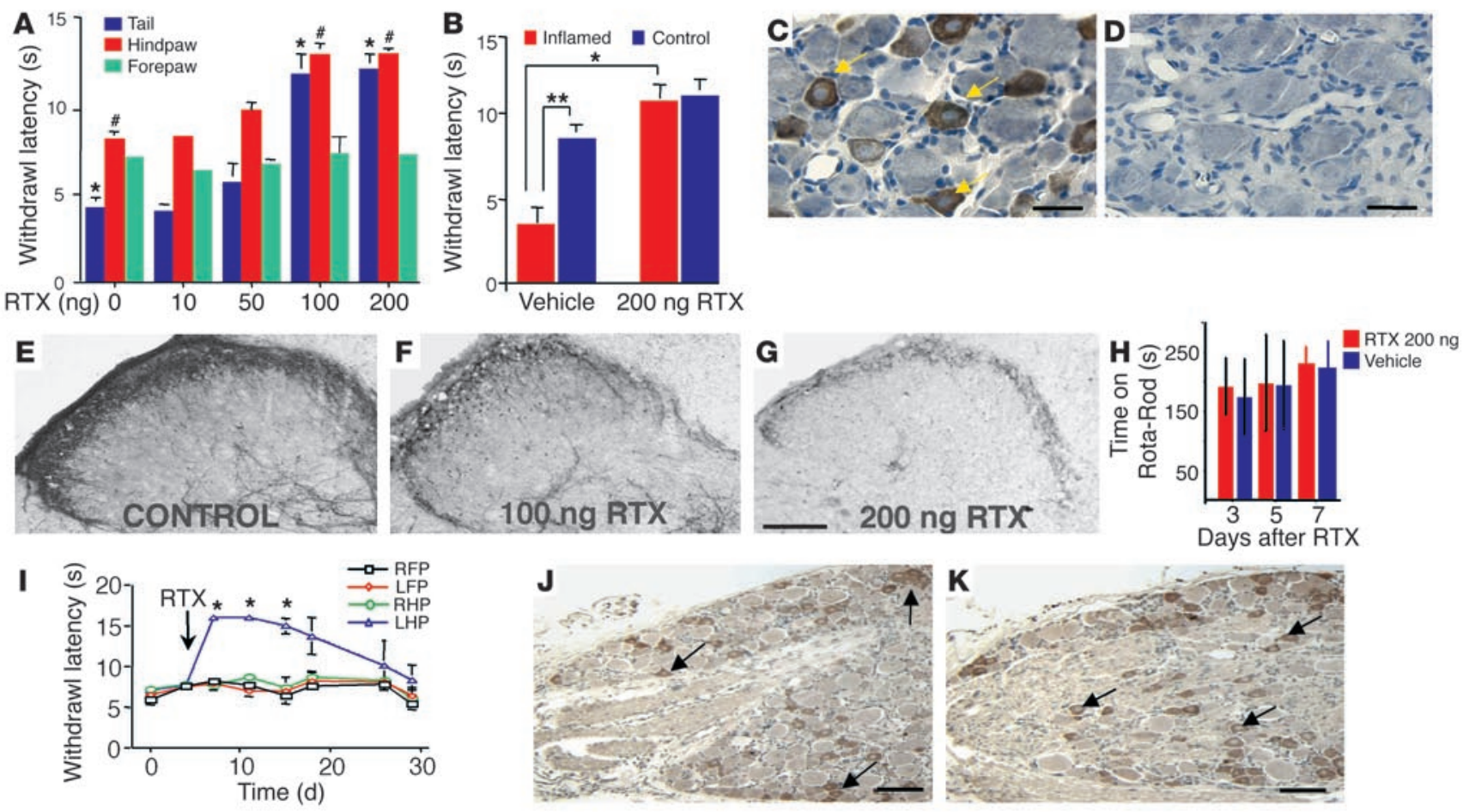

\section{Figure 3}

Intrathecal (multiganglionic) or peripheral RTX attenuates thermal nociception and inflammatory hyperalgesia. (A) Dose-response to lumbar intrathecal administration of $\operatorname{RTX}\left(n=50,10\right.$ per group; $\left.{ }^{*}, \#<0.05\right)$. Doses lower than 50 ng were without effect. Robust analgesia was obtained for the tail and hindpaws at doses of 100 and $200 \mathrm{ng}$. (B) Carrageenan inflammatory hyperalgesia $\left(n=4\right.$ per group; $\left.{ }^{* *} P<0.05\right)$ is reversed after $200 \mathrm{ng}$ intrathecal $\operatorname{RTX}\left({ }^{\star} P<0.05\right)$. No analgesic effect was seen in forepaws $(\mathbf{A})$, correlating with retention of TRPV1-IR neurons in the cervical ganglia (C) and their loss in lumbosacral ganglia (D). Dose-related reduction of CGRP-IR, a neuropeptide expressed by nociceptive afferent terminals, in the lumbar spinal cord at 3 days after administration of RTX (E-G). No significant effect on locomotor performance occurs as examined using an accelerating Rota-Rod (H). Peripheral left hindpaw (LHP) administration of $100 \mathrm{ng}$ RTX yields unilateral and reversible (about 20 days) thermal analgesia ("ANOVA with Scheffe's post hoc test; $P<0.005)(n=5$ per group). RHP, right hindpaw; FP, forepaw. (I) Retention of TRPV1IR neurons (arrows) in L5 DRGs from RTX-injected (J) and noninjected (K) hindpaws. Cell counts of TRPV1-IR perikarya showed no significant alteration between left and right lumbar ganglia (see Results). Bars: $50 \mu \mathrm{m}$ (C and D); $100 \mu \mathrm{m}$ (J and K); $300 \mu \mathrm{m}$ (E-G).

and (b) the fact that neoplastic advancement did not diminish the RTX-induced analgesia.

Comparative histology of rat and canine sensory ganglia demonstrated that RTX eliminated many small-diameter neuronal cell bodies in both species, leaving no visible damage to surrounding neuropil (Figure 4, B-G). The early morphological signs of specific cell deletion were studied in rats sacrificed at 6,12 , and 24 hours. During this period, nuclear envelope ruffling and displacement of the nucleus in specific ganglion neurons were noted, indicating early stages of neuronal damage (Figure 4, B and C). In dogs, satellite cell activation and neuronophagia occurred around the dying neurons 3 weeks after intrathecal RTX treatment, progressing into characteristic nodules of Nageotte, which have been described in human diseases with pathological sensory aspects (Figure 4, D and E) (32). After 1 year, retention of the large-size neurons and deposition of fine ECM inside satellite cell rosettes were observed in rat ganglia without abnormal scar formation (Figure 4, F and G).

Effect of RTX on human DRG neurons. The ligand-activated cell deletion approach for pain control is based on the enriched expression of TRPV1 in a subset of sensory ganglion neurons. Immunocytochemistry and live-cell imaging were performed to determine expression of TRPV1 in adult human sensory ganglia and demonstrate whether selectivity can be expected upon administration into humans (Figure 5). While the cloned human TRPV1 has been studied in heterologous expression systems (33) and in an immortalized human neuronal cell line (34), there are no data on the physiological response and selectivity of primary human DRG neurons to stimulation by vanilloids. Figure 5 depicts the structure of a normal adult human DRG, and immunofluorescence (green) reveals human TRPV1-expressing neurons. There are "empty" lacunae surrounded by Hoechst $33342^{+}$satellite cells (blue) that are occupied by neurons, which are negative for human TRPV1 expression.

RTX opens the human TRPV1 ion channel and increases the $\left[\mathrm{Ca}^{2+}\right]_{\mathrm{i}}$ inside human TRPV1+ DRG neurons. This change of $\left[\mathrm{Ca}^{2+}\right]_{\mathrm{i}}$ can be detected with Fluo-4 AM, which changes its fluorescence emission intensity according to the $\left[\mathrm{Ca}^{2+}\right]_{i}$ (Figure 5, E-G). Fluo-4 AM imaging clearly distinguishes two populations of neurons: vanilloid sensitive and vanilloid insensitive. The $\left[\mathrm{Ca}^{2+}\right]_{\mathrm{i}}$ in responding human neurons (Figure 5, E-G; cells 1-4) remains high for a prolonged period of time, suggesting a damaged, incapacitated state and imminent cell death. The images and line traces from nonresponding cells (Figure 5, E-G; cells 5-7) are also important, since they demonstrate the selectivity of the RTX treatment and the lack of toxicity to the nonresponding neurons or satellite cells. 

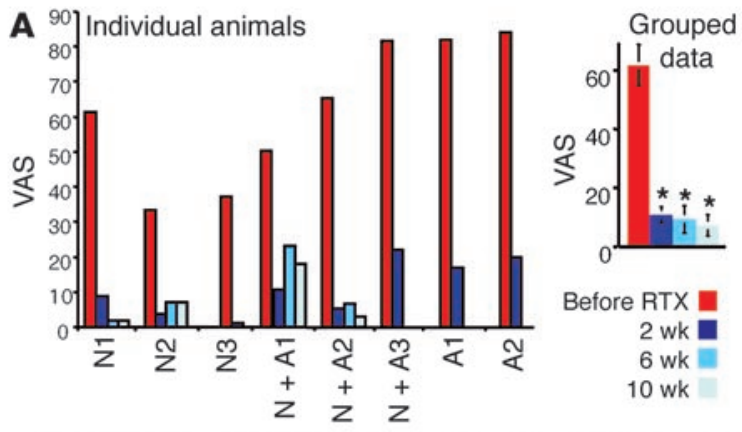

Before RTX
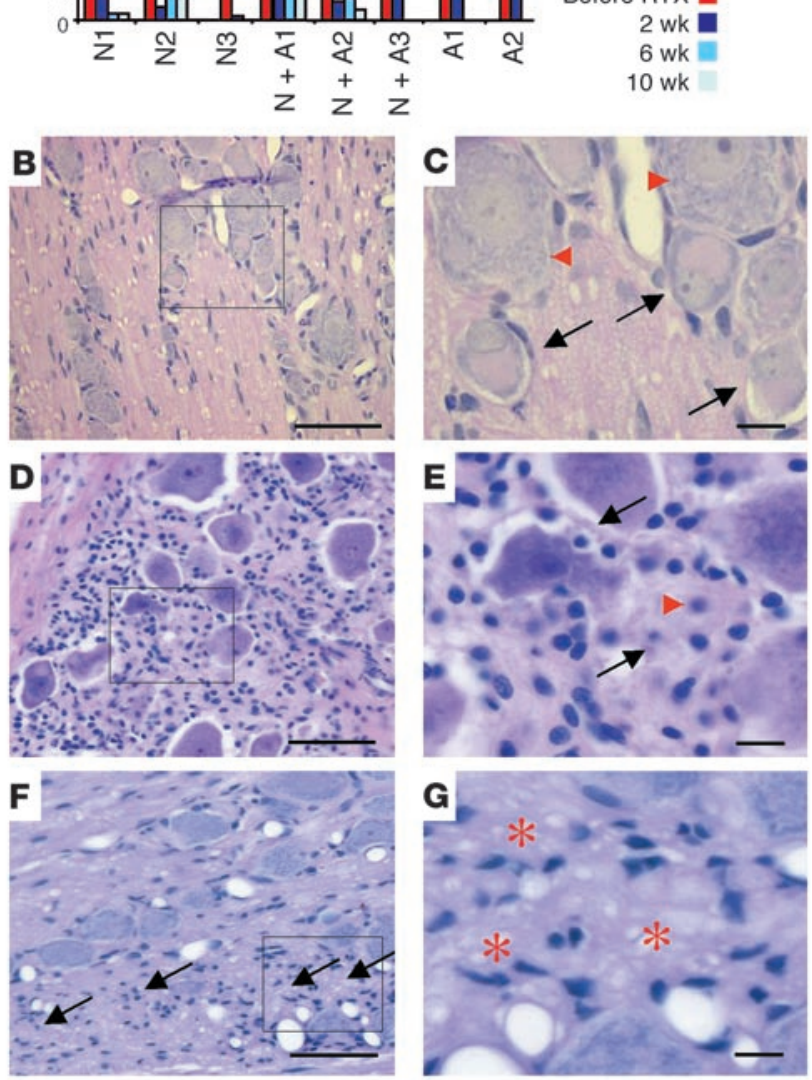

\section{Discussion}

These data demonstrate the crucial and necessary role that TRPV1 ${ }^{+}$ neurons play in supporting nociception arising from inflammatory hyperalgesia, osteoarthritis, and cancer, and how this can be exploited to produce a new class of pain treatments. The RTX intervention removes the "C-fiber line of communication" but is not equivalent to drugs that block one of the multiple neuroactive substances these neurons release or respond to (e.g., substance P, CGRP, glutamate, bradykinin, prostaglandins). The diversity of transmitters and receptors involved in pain generation suggests that blocking only one element may not yield effective analgesia $(6,12,35)$. In contrast, eliminating the participation of TRPV1-expressing neurons at either the nerve ending or the perikarya reveals the algesic profile of these neurons and their potential as pharmacological targets. Furthermore, the present chemical cellular knockout is not equivalent to removal of the TRPV1 molecule in TRPV1 KO mice $(25,26)$, although the behavioral repertoire seen with RTX-neuronal deletion is quite similar and reinforces the idea that TRPV1 is a key molecule in transduction of nociceptive stimuli.

RTX-induced $\mathrm{Ca}^{2+}$ cytotoxicity is the proposed mechanism for therapeutic cell deletion (16). Experiments in vivo demonstrate the behavioral effectiveness of RTX, and immunohistochemistry con-

\section{Figure 4}

RTX analgesia in naturally occurring neoplasms or osteoarthritis in the dog and morphological aspects of cell deletion in dog DRG and rat TG over time. (A) Intrathecal RTX reduced pain reports by the owners using a VAS in neoplastic (N), arthritic (A), and neoplastic plus arthritic $(\mathrm{N}+\mathrm{A})$ dogs $(n=8)$. Bars represent individual animals; inset represents summed data ('ANOVA with Scheffe's post hoc test; $P<0.05$ ). (B) High-power $(\times 40)$ image of an adult rat TG, 24 hours after 200 ng intraganglionic RTX administration, shows grossly normal appearance. (C) The enlargement reveals swollen eosinophilic cytoplasm and dislocated nuclei with partial wavy pattern of the nuclear membrane in many small- to medium-sized neurons (black arrows), characteristic of calcium cytotoxicity. Large neurons (red arrowheads) are normal. (D) DRG from an adult dog 21 days after intrathecal RTX injection shows patchy proliferation of satellite cells. (E) At higher magnification, neuronophagia is evident (black arrows). A necrotic neuron in which the nuclear membrane is faintly visible around a spot of condensed nuclear material is also visible (red arrowhead). By 30 days in dog ganglia, damaged and dead neurons are replaced by proliferating satellite cell colonies called nodules of Nageotte, which can be seen as a ball of proliferating cells (not shown). (F) One year after RTX treatment, the rat TG shows extended areas of acellular eosinophilic fields surrounded by rosetting satellite cells, indicated by red asterisks at higher magnification (G). There is no evidence of excessive, distorting glial proliferation. Bars: $100 \mu \mathrm{m}$ (B, D, and F); $25 \mu \mathrm{m}$ (C, E, and G).

firms that RTX deletes TRPV1+ ${ }^{+}$neurons. Our results are consistent with earlier in vitro observations of CAP/RTX-mediated damage and elimination of cultured rat DRG neurons or cells ectopically expressing TRPV1 and do not appear to support a receptor desensitization mechanism for the analgesic actions $(21,36-39)$. The potency of RTX yields a duration and degree of intracellular calcium elevation that makes it fundamentally more efficacious for cell deletion in adult animals than CAP, which while highly algesic (40), is a readily reversible agonist of TRPV1 $(16,41,42)$.

The data presented here suggest that cellular excision by "molecular neurosurgery" would confer several advantages over neurosurgical, chemical (phenol, ethanol), or radiofrequency neuroablative techniques in terms of selectivity, efficacy, and lack of side effects. RTX removes one class of neurons, whereas the other treatments lack sensory modality selectivity, and, if motor axons are compromised, bring about loss of muscular control. Conventional ablative approaches often yield incomplete relief or evolve into a deafferentation pain syndrome (43). Indiscriminate destruction of ganglionic neurons produces extensive proliferation of glia, inflammatory, and connective tissue cells, leading to scar formation and neuropathic sensory problems $(43,44)$. RTX excises the TRPV1 ${ }^{+}$neurons in such a way that formation of abnormal anatomical structures is circumvented. In RTX-treated animals, the lack of chronic behavioral maladaptations also may be due to retention of afferents not expressing TRPV1, incomplete removal of C-fiber neurons, and the fact that C-fiber primary afferents ascend and descend five or more dermatomes (45). All of these influences may prevent trophic disturbances and secondary neuronal alterations due to loss of synaptic contacts. Furthermore, TRPV1 cell deletion is not equivalent to peripheral nerve axotomy. Formerly, researchers had thought that rearrangement of A- $\beta$ fibers occurred upon damage to peripheral nerve, which stimulated collateral sprouting of A-beta fibers into the superficial dorsal horn and conferred mechanosensitive allodynia and abnormal behaviors in animals (autotomy) (28, 

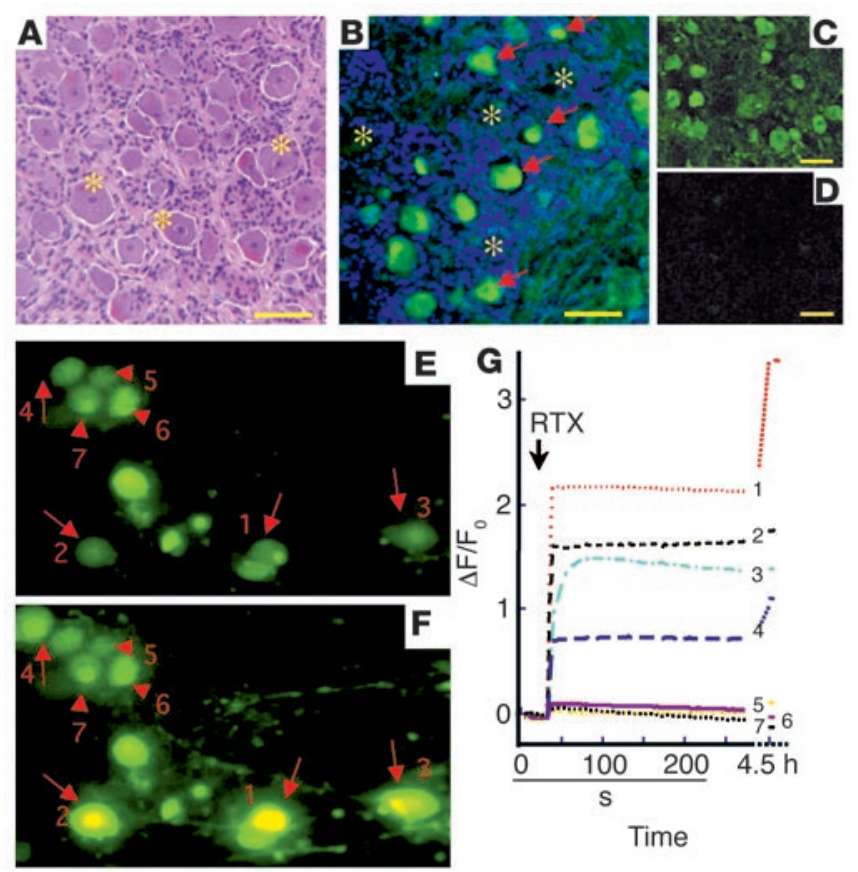

46). Recent evidence suggests that sprouting is substantially less than originally reported $(47,48)$.

Deletion of nociceptors is especially effective in blocking inflammatory hyperalgesia in rats and clinical manifestations of pain in dogs. However, the loss of the TRPV1+ nociceptors does not eliminate all types of pain sensation. Many animals remained responsive to thermal stimuli, and not all went to the behavioral cutoff. No significant alteration in response to noxious mechanical sensation was detected, as was also noted with intrathecal administration of CAP (37), indicating the presence of a population of high-threshold mechanoreceptive afferents that do not express TRPV1. No tendency to increased sensitivity to mechanical stimuli (von Frey hairs or withdrawal duration after pinprick) developed, suggesting that TRPV1-neuronal deletion, as performed here, does not induce a neuropathic pain state (49).

The anatomically directed nature of RTX administration allows the treatment to be tailored to the site (or sites) of nociceptive generation using interventional procedures with either a reversible or irreversible outcome as the therapeutic goal. Routes of administration can include single or multiple intraganglionic injections, intrathecal application for disseminated pain problems, or injections into peripheral sites. In all cases, the same principle of receptor-mediated $\mathrm{Ca}^{2+}$ cytotoxicity is operative (50), but the present data show that the distal injections do not eliminate the TRPV1-expressing DRG neurons, and therefore the analgesic effect is transient. The peripheral data support a spatially delimited Wallerian-like degeneration occurring locally and specifically in vanilloid-sensitive fibers $(31,51)$. Because the damaged ending remains connected to an intact cell body, the ending reinnervates the skin, a process that requires approximately 20 days in the present study. This reinnervation is consistent with studies of topically applied CAP (52) and recent data using RTX (31). Myelinated fibers are not affected as was reported earlier with CAP (53). The extent of the reversible degeneration correlates with the RTX application. While the intraganglionic and periph-

\section{Figure 5}

Human DRG neurons show selective sensitivity to RTX treatment. (A) H\&E section, adult human DRG (*large-sized neurons). (B-D) Green immunofluorescence human TRPV1-IR neurons (arrows), contrasted with satellite cell nuclei (blue). (C) TRPV1-IR (green) is adsorbed by the peptide antigen (D). Selective response of human embryonic DRG neurons to RTX assessed by increases in $\left[\mathrm{Ca}^{2+}\right]_{i}$ using Fluo-4 AM imaging ( $E$ and $\mathbf{F})$. Arrows indicate responding neurons, and arrowheads indicate nonresponding neurons. $E$ shows the baseline fluorescence depicted as near 0 for the first 30 seconds of the normalized data in the graph $(\mathbf{G})$. In $\mathbf{F}$, the transmembrane calcium flux leads to increases in intracellular calcium in specific neurons, which become brightly fluorescent. Magnification $\times 200$. (G) Traces of individual cells showing a substantial and abrupt response in vanilloid-sensitive sensory neurons (traces $1-4)$. Elevation of $\left[\mathrm{Ca}^{2+}\right]_{\mathrm{i}}$ for a prolonged period of time is suggestive of imminent cell death in cells responding to vanilloid treatment. The RTX effect is clearly selective; nonresponding cells (traces 5-7) maintain normal calcium levels. Increase in normalized fluorescence intensity (numbered $\Delta F / F_{0}$ traces refer to cells in $E$ and F). Bars: $100 \mu \mathrm{m}$.

eral injections are well localized, the intrathecal and intracisternal routes have the potential to reach the brain via the cerebrospinal fluid. We observed no behavioral abnormalities in RTX-treated rats. In the dogs, even with intracisternal administration, there were no reports of abnormal personality changes. RNA blot (14) and our own immunohistochemical studies have not detected TRPV1 expression in brain regions or immunopositive neurons. The lack of behavioral alterations suggests that, despite reports of CAP actions $(54,55)$, RTX-binding sites (56), or TRPV1 expression (57), higher brain centers are not substantially affected upon intracisternal or intrathecal administration.

Elements of the approach outlined here are scattered in the literature but have not been integrated into an explicit therapeutic intervention for several possible reasons: (a) early studies used CAP, which is less effective for cell deletion; (b) vanilloid agonists are generally thought of as algesic agents; and (c) the proposed mechanism(s) of action were either vaguely defined or inconsistent to form a basis for human clinical trials $(21,36-38,40,41,52$, 58). In contrast, the direct observation of RTX action, in real time, on in vitro DRG neurons or TRPV1-expressing cells provided a visually appreciable mechanism for therapeutic application (16).

The present experiments address an additional key issue in analgesia research, which is the predictive relationship between evaluation of new drugs in experimental models and their eventual performance in human clinical pain, where they are frequently ineffective or only minimally so. The canine model we introduce provides a transitional bridge between nonclinical rodent data and eventual human trials, and reinforces the idea that a treatment will be safe and effective. The canine study also highlights the need for better measurement tools for large-animal studies. The results with RTX were robust enough to be easily rated by the human observers; however, new methods to obtain objective, meaningful measurements should be developed. The selectivity of RTX in live-cell imaging of human DRG neurons provides a second type of translational observation between basic in vitro observations 
and human clinical application. These data represent a progressive series of steps from experimental rodent models to clinical veterinary models, to human cells that explore the clinical potential of selective nociceptive neuronal deletion for pain control. The celldeletion approach may provide an important alternative to present treatments, and its eventual use will help to improve the quality of life in patients with unrelenting pain.

\section{Methods}

Animals. Procedures for rat studies followed the NIH Guidelines for the Care and Use of Laboratory Animals, and were approved by the National Institute of Dental and Craniofacial Research Animal Care and Use Committee. A protocol to treat dogs with RTX was approved by the University of Pennsylvania School of Veterinary Medicine Institutional Animal Care and Use Committee prior to the start of the study.

Trigeminal microinjection. Male Sprague-Dawley rats (300 g, $n=54$ ) were anesthetized with ketamine/xylazine and mounted in a stereotaxic frame. A 10- $\mu$ l Hamilton syringe (Hamilton Company, Reno, Nevada, USA) was positioned at $2.5 \mathrm{~mm}$ posterior and $1.5 \mathrm{~mm}$ lateral to the bregma. The skull was drilled, and the needle was advanced until it gently touched the base of the skull; the needle was retracted $0.1 \mathrm{~mm}$, and RTX (20 or $200 \mathrm{ng}$ ) was injected in a volume of $2 \mu \mathrm{l}$ over 10 minutes. A pharmacological-grade preparation of RTX was formulated as follows: PBS, $\mathrm{pH}$ 7.2, containing $0.1 \mu \mathrm{g} / \mu \mathrm{l}$ RX, $0.05 \%$ ascorbic acid, and $7 \%$ Tween 80. Vehicle contained no RTX. The Pharmaceutical Development Section, Clinical Center, NIH, performed characterization of this preparation for drug purity, stability, presence of endotoxin, and microbiological contamination.

Immunohistochemistry. Tissues were fixed in $10 \%$ buffered formalin for 24 hours and processed in paraffin. Five $\mu \mathrm{m}$ sections were stained with $\mathrm{H} \& \mathrm{E}$ or used for immunohistochemistry as described (31). Primary antibodies used were anti-rat TRPV1, 1:1,000 (Affinity Bioreagents Inc., Golden, Colorado, USA), CGRP, 1:2,500, and mouse monoclonal N52 (1:2,000) (Chemicon International, Temecula, California, USA).

Histology and immunohistochemistry of adult human DRG. Tissue for neuroanatomical studies was obtained under a protocol with the Delaware Valley Kidney 1 Human Organ Donor Program. DRGs were removed within 1 hour of cessation of perfusion, and either specimens were paraffin-processed or frozen sections were cut for histochemistry or immunohistochemistry, respectively. Human TRPV1-specific antibody (PA1-748; Affinity BioReagents Inc.) was used at a dilution of 1:500 and incubated overnight at $4^{\circ} \mathrm{C}$ visualized with FITC-conjugated AffiniPure Donkey Anti-Rabbit IgG (711-095-152; Jackson ImmunoResearch Laboratories Inc., West Grove, Pennsylvania, USA) and counterstained with Hoechst 33342 dye (H3570; Molecular Probes Inc., Eugene, Oregon, USA). Control sections for assessment of nonspecific binding were processed in an identical way, except that primary antibody steps contained $2.5 \mu \mathrm{g}$ peptide antigen $/ \mathrm{ml}$.

Human primary DRG culture. Human DRG tissue was obtained from Advanced Bioscience Resources Inc. (Alameda, California, USA). Ganglia were collected in cold Lebowitz medium (Invitrogen Corp., Carlsbad, California, USA). After digestion with $0.125 \%$ trypsin at $37^{\circ} \mathrm{C}$ for 25 minutes, cells were seeded on coverslips coated with poly-D-lysine. Culture medium contained DMEM with $20 \mathrm{mM}$ HEPES, 7.5\% FBS, 7.5\% horse serum, and 50 $\mu \mathrm{g} / \mathrm{ml}$ nerve growth factor. After the first day, $5 \mathrm{mg} / \mathrm{ml}$ uridine,
$2 \mathrm{mg} / \mathrm{ml}$ FUDR (Sigma-Aldrich, St. Louis, Missouri, USA) was added to inhibit nonneuronal cell division.

Fluorescent microscopy and calcium imaging. Fluorescent imaging was performed with an Olympus IX 70 microscope equipped with the CARV confocal imaging system (Atto Bioscience Inc., Rockville, Maryland, USA). Calcium imaging with Fluo-4 AM (F14201; Molecular Probes Inc.) was performed in an open imaging chamber. Calcium concentration was analyzed using the AttoVision (Atto Bioscience Inc.) and KaleidaGraph (Synergy Software, Reading, Pennsylvania, USA) software packages.

$R N A$ extraction and RT-PCR. Dissected ganglia were immediately frozen at $-80^{\circ} \mathrm{C}$. Total RNA was extracted and treated with RNase-free DNase; 5 ng were then reverse transcribed and amplified over the linear range (26 cycles) using the Access RT-PCR System (Promega, Madison, Wisconsin, USA). The rat TRPV1specific primers, TG-5'-AAGCGCACCCTGAGCTTCTC and TG3'-CTCGAGTGCTTGCATCCCTC yielded a 100-bp product; the GAPDH-specific primers, 5'-ACCACAGTCCATGCCATCAC and 3'-TCCACCACCCTGTTGCTGTA, generated a 452-bp RT-PCR product. DNAs were separated by electrophoresis in $1 \%$ agarose gels, stained, and quantified by densitometry.

Intrathecal injection of rats. Animals $(n=58)$ were anesthetized with isoflurane, and RTX (10-200 ng in $30 \mu$ l over 10 minutes) or vehicle was administered by inserting a 20 -gauge guide catheter into the L4/L5 interspace. A PE-10 catheter was advanced $1 \mathrm{~cm}$ into the intrathecal space. The criterion for the intrathecal placement was withdrawal of clear cerebrospinal fluid into the catheter.

Intraplantar RTX injection. Rats $(n=20)$ were injected with vehicle or RTX $(100 \mu \mathrm{l})$ over a concentration range of 1,10 , and $100 \mu \mathrm{g} /$ $\mathrm{ml}$. A dose response was observed, and time course data for the $1-\mu \mathrm{g} / \mathrm{ml}$ dose are shown (Figure $3 \mathrm{I})$. Cell counts were made on three sections ipsilateral and three sections contralateral from the L5 ganglia from three rats.

Sensory and physiological tests. The afferent functions of the trigeminal nerve were tested in 30 rats after TG microinjection of RTX using the CAP-induced eye-wipe response. A stock solution of $5 \%$ CAP was made in $75 \%$ ethanol, $0.05 \%$ ascorbic acid (Sigma-Aldrich). After dilution to $0.01 \%$, CAP with saline (50 $\mu l)$ was dropped into the cornea, and eye wipes were counted for 1 minute. A separate group of seven rats was tested over the course of a year.

Efferent functions of the trigeminal nerve were tested in rats $(n=7)$ at 7 days after injection. Rats were anesthetized with ketamine/xylazine and hair removed with Nair (Carter-Wallace Inc., New York, New York, USA). After washing thoroughly with water, $5 \%$ CAP cream, containing $0.05 \%$ ascorbic acid, was applied evenly to the entire head and shoulder region. After 15 minutes, the left femoral vein was catheterized and a 0.5 - $\mathrm{ml}$ bolus of Evans Blue (Sigma-Aldrich) $(20 \mathrm{mg} / \mathrm{kg}$ in saline) was injected.

Radiant-heat thermal testing was performed on unrestrained rats $(n=58)$ according to previously published procedures (23, 24). The endpoint recorded was latency (seconds) to paw withdrawal. Thermal intensity was set to produce a withdrawal latency of 8-10 seconds. A 10 -second withdrawal latency corresponds to a temperature at the surface of the paw of $45.2^{\circ} \mathrm{C}(23)$. Mechanical sensory stimulation included a pinch with toothed forceps, the application of graded von Frey filaments, and assessment of pinprick hyperalgesia as described previously (27). Rats treated 3 and 10 days and 1 year ( $n=4$ per group) prior to mechanosensory testing and a group of untreated controls $(n=4)$ were examined. 
Reduction in inflammatory hyperalgesia was tested by radiant thermal heat 5 days after intrathecal injection of either vehicle or RTX (200 ng; $n=8$, four to a group). Rats were lightly anesthetized with isoflurane, and the plantar surface of the left hindpaw was injected with $100 \mu l$ 4\% carrageenan (C-3889; Sigma-Aldrich), dissolved in saline.

Assessment of locomotion and proprioception was performed in the intrathecally injected (vehicle and RTX) rats on an accelerating Rota-Rod (4-40 rpm in 5 min; model 7750; Ugo Basile Biological Research Apparatus, Comerio, Italy).

Intrathecal injection of dogs. We recruited dogs having advanced cancer or osteoarthritis $(n=8)$, whose owners brought them to the veterinary clinic for evaluation because of pain not well controlled by current medications. This was an open-design study, both for ethical reasons and because of the very prolonged duration of action of a single intrathecal dose of RTX, which we hypothesized would provide sustained improvement in activity and animal personality issues, thereby outlasting a 'placebo effect' in third-party observers.

Dogs were maintained under general anesthesia with isoflurane and oxygen. The cisterna magna was penetrated with a 20 -gauge $4-\mathrm{cm}$ spinal needle. When the needle is properly placed, the cerebrospinal fluid flows freely. A single dose of $1 \mu \mathrm{g} / \mathrm{kg}$ of RTX was injected over 10 minutes, followed by $0.2 \mathrm{ml}$ of sterile saline; any arousing effects were controlled with intravenous fentanyl.
VAS ratings. To assess the effect of a single dose of intrathecal RTX, owners were asked to rate their dogs' pain before and after treatment, using a $100-\mathrm{mm}$ VAS, with zero designated as no pain and 100 the worst pain imaginable. Ratings were obtained before injection, and at 2, 6, and 10 weeks after injection. (The lack of data in several cases results from euthanasia following progression or complications of the main pathology.)

\section{Acknowledgments}

This research was supported by a Bench-to-Bedside Initiative from the Clinical Center, NIH, and the Division of Intramural Research, National Institute of Dental and Craniofacial Research. We thank Chino Anyanwu, Ruth Yaskovich, and Joseph Nezgoda for their outstanding technical support. We also thank Susan Hoover and Jeffrey Cohen for their help with the human DRG cultures.

Received for publication November 3, 2003, and accepted in revised form February 25, 2004.

Address correspondence to: Michael J. Iadarola, National Institute of Dental and Craniofacial Research, 49 Convent Drive, Bethesda, Maryland 20892, USA. Phone: (301) 496-2758; Fax: (301) 401-0667; E-mail:miadarola@dir.nidcr.nih.gov.
1. Bernabei, R., et al. 1998. Management of pain in elderly patients with cancer. SAGE Study Group. Systematic Assessment of Geriatric Drug Use via Epidemiology. JAMA. 279:1877-1882.

2. Cleeland, C.S., et al. 1994. Pain and its treatment in outpatients with metastatic cancer. N. Engl. J. Med. 330:592-596.

3. Wolfe, J., et al. 2000. Symptoms and suffering at the end of life in children with cancer. N. Engl. J. Med. 342:326-333.

4. Eddy, N.B., and May, E.L. 1973. The search for a better analgesic. Science. 181:407-414.

5. Nitu, A.N., Wallihan, R., Skljarevski, V., and Ramadan, N.M. 2003. Emerging trends in the pharmacotherapy of chronic pain. Expert Opin. Investig. Drugs. 12:545-559.

6. Scholz, J., and Woolf, C.J. 2002. Can we conquer pain? Nat. Neurosci. 5 (Suppl):1062-1067.

7. Cowan, J.D., and Walsh, D. 2001. Terminal sedation in palliative medicine-definition and review of the literature. Support Care Cancer. 9:403-407.

8. Cowan, J.D., and Palmer, T.W. 2002. Practical guide to palliative sedation. Curr. Oncol. Rep. 4:242-249.

9. Harrison, P. 2001. Update on pain management for advanced genitourinary cancer. J. Urol. 165:1849-1857; discussion 157-158.

10. Foley, K.M., and Gelband, H. 2001. Improving palliative care for cancer. National Academy Press, Washington DC, USA. 325 pp.

11. Smith, T.J., et al. 2002. Randomized clinical trial of an implantable drug delivery system compared with comprehensive medical management for refractory cancer pain: impact on pain, drug-related toxicity, and survival. J. Clin. Oncol. 20:4040-4049.

12. Richardson, J.D., and Vasko, M.R. 2002. Cellular mechanisms of neurogenic inflammation. J. Pharmacol. Exp. Ther. 302:839-845.

13. Tominaga, M., et al. 1998. The cloned capsaicin receptor integrates multiple pain-producing stimuli. Neuron. 21:531-543.

14. Caterina, M.J., et al. 1997. The capsaicin receptor: a heat-activated ion channel in the pain pathway. Nature. 389:816-824.

15. Szallasi, A., and Blumberg, P.M. 1989. Resiniferatoxin, a phorbol-related diterpene, acts as an ultrapotent analog of capsaicin, the irritant constituent in red pepper. Neuroscience. 30:515-520.

16. Olah, Z., et al. 2001. Ligand-induced dynamic membrane changes and cell deletion conferred by vanilloid receptor 1. J. Biol. Chem. 276:11021-11030.

17. Jancso, N., Jancso-Gabor, A., and Szolcsanyi, J. 1967. Direct evidence for neurogenic inflammation and its prevention by denervation and by pretreatment with capsaicin. Br. J. Pharmacol. 31:138-151.

18. Holzer, P. 1998. Neurogenic vasodilatation and plasma leakage in the skin. Gen. Pharmacol. 30:5-11.

19. Bhave, G., et al. 2002. cAMP-dependent protein kinase regulates desensitization of the capsaicin receptor (VR1) by direct phosphorylation. Neuron. 35:721-731.

20. Liu, L., and Simon, S.A. 1998. The influence of removing extracellular $\mathrm{Ca}^{2+}$ in the desensitization responses to capsaicin, zingerone and olvanil in rat trigeminal ganglion neurons. Brain Res. 809:246-252.

21. Holzer, P. 1991. Capsaicin: cellular targets, mechanisms of action, and selectivity for thin sensory neurons. Pharmacol. Rev. 43:143-201.

22. Price, T.J., Helesic, G., Parghi, D., Hargreaves, K.M., and Flores, C.M. 2003. The neuronal distribution of cannabinoid receptor type 1 in the trigeminal ganglion of the rat. Neuroscience. 120:155-162.

23. Hargreaves, K., Dubner, R., Brown, F., Flores, C., and Joris, J. 1988. A new and sensitive method for measuring thermal nociception in cutaneous hyperalgesia. Pain. 32:77-88.

24. Iadarola, M.J., Douglass, J., Civelli, O., and Naranjo, J.R. 1988. Differential activation of spinal cord dynorphin and enkephalin neurons during hyperalgesia: evidence using cDNA hybridization. Brain Res. 455:205-212.

25. Davis, J.B., et al. 2000. Vanilloid receptor-1 is essential for inflammatory thermal hyperalgesia. Nature. 405:183-187.

26. Caterina, M.J., et al. 2000. Impaired nociception and pain sensation in mice lacking the capsaicin receptor [see comments]. Science. 288:306-313.

27. Benoliel, R., et al. 1999. Actions of intrathecal diphtheria toxin-substance $P$ fusion protein on models of persistent pain. Pain. 79:243-253.

28. Zimmermann, M. 2001. Pathobiology of neuropathic pain. Eur. J. Pharmacol. 429:23-37.
29. Kauppila, T. 1998. Correlation between autotomybehavior and current theories of neuropathic pain. Neurosci. Biobehav. Rev. 23:111-129.

30. Macrae, W.A. 2001. Chronic pain after surgery. $B r$. J. Anaesth. 87:88-98.

31. Neubert, J.K., et al. 2003. Peripherally induced resiniferatoxin analgesia. Pain. 104:219-228.

32. Graham, D.I., and Lantos, P.L. 1997. Greenfield's Neuropathology. 6th edition. Volume 2. Arnold. New York, New York, USA. Oxford University Press. London, United Kingdom. 395 pp.

33. Smart, D., et al. 2001. Characterisation using FLIPR of human vanilloid VR1 receptor pharmacology. Eur. J. Pharmacol. 417:51-58.

34. Raymon, H.K., et al. 1999. Immortalized human dorsal root ganglion cells differentiate into neurons with nociceptive properties. J. Neurosci. 19:5420-5428.

35. Dionne, R.A., et al. 1998. The substance P receptor antagonist CP-99,994 reduces acute postoperative pain. Clin. Pharmacol. Ther. 64:562-568.

36. Jancso, G., and Kiraly, E. 1981. Sensory neurotoxins: chemically induced selective destruction of primary sensory neurons. Brain Res. 210:83-89.

37. Yaksh, T.L., Farb, D.H., Leeman, S.E., and Jessell, T.M. 1979. Intrathecal capsaicin depletes substance $\mathrm{P}$ in the rat spinal cord and produces prolonged thermal analgesia. Science. 206:481-483.

38. Nagy, J.I., Emson, P.C., and Iversen, L.L. 1981. A reevaluation of the neurochemical and antinociceptive effects of intrathecal capsaicin in the rat. Brain Res. 211:497-502.

39. Appendino, G., and Szallasi, A. 1997. Euphorbium: modern research on its active principle, resiniferatoxin, revives an ancient medicine. Life Sci. 60:681-696.

40. Iadarola, M.J., et al. 1998. Neural activation during acute capsaicin-evoked pain and allodynia assessed with PET. Brain. 121:931-947.

41. Jeftinija, S., Liu, F., Jeftinija, K., and Urban, L. 1992. Effect of capsaicin and resiniferatoxin on peptidergic neurons in cultured dorsal root ganglion. Regul. Pept. 39:123-135.

42. Maggi, C.A., et al. 1990. Similarities and differences in the action of resiniferatoxin and capsaicin on central and peripheral endings of primary sensory neurons. Neuroscience. 37:531-539. 
43. Sweet, W.H. 1984. Deafferentation pain after posterior rhizotomy, trauma to a limb, and herpes zoster. Neurosurgery. 15:928-932.

44. Taha, J.M., and Tew, J.M., Jr. 1996. Comparison of surgical treatments for trigeminal neuralgia: reevaluation of radiofrequency rhizotomy. Neurosurgery. 38:865-871.

45. Traub, R.J., Iadarola, M.J., and Ruda, M.A. 1989. Effect of multiple dorsal rhizotomies on calcitonin gene-related peptide-like immunoreactivity in the lumbosacral dorsal spinal cord of the cat: a radioimmunoassay analysis. Peptides. 10:979-983.

46. Woolf, C.J., Shortland, P., and Coggeshall, R.E. 1992. Peripheral nerve injury triggers central sprouting of myelinated afferents. Nature. 355:75-78.

47. Santha, P., and Jancso, G. 2003. Transganglionic transport of choleragenoid by capsaicin-sensitive C-fibre afferents to the substantia gelatinosa of the spinal dorsal horn after peripheral nerve section. Neuroscience. 116:621-627.

48. Bao, L., et al. 2002. Peripheral axotomy induces only very limited sprouting of coarse myelinated afferents into inner lamina II of rat spinal cord. Eur. J. Neurosci. 16:175-185.

49. Pan, H.L., Khan, G.M., Alloway, K.D., and Chen, S.R 2003. Resiniferatoxin induces paradoxical changes in thermal and mechanical sensitivities in rats: mechanism of action. J. Neurosci. 23:2911-2919.

50. Caudle, R.M., et al. 2003. Resiniferatoxin-induced loss of plasma membrane in vanilloid receptor expressing cells. Neurotoxicology. 24:895-908.

51. LaMotte, R.H., Lundberg, L.E., and Torebjork, H.E. 1992. Pain, hyperalgesia and activity in nociceptive $\mathrm{C}$ units in humans after intradermal injection of capsaicin. J. Physiol. 448:749-764.

52. Nolano, M., et al. 1999. Topical capsaicin in humans: parallel loss of epidermal nerve fibers and pain sensation. Pain. 81:135-145.

53. Ferrell, W.R., Lam, F.Y., and Montgomery, I. 1992. Differences in the axon composition of nerves supplying the rat knee joint following intra-articular injection of capsaicin. Neurosci. Lett. 141:259-261.
54. Sasamura, T., Sasaki, M., Tohda, C., and Kuraishi, Y. 1998. Existence of capsaicin-sensitive glutamatergic terminals in rat hypothalamus. Neuroreport. 9:2045-2048.

55. Marinelli, S., Vaughan, C.W., Christie, M.J., and Connor, M. 2002. Capsaicin activation of glutamatergic synaptic transmission in the rat locus coeruleus in vitro. J. Physiol. 543:531-540.

56. Szabo, T., Biro, T., Gonzalez, A.F., Palkovits, M., and Blumberg, P.M. 2002. Pharmacological characterization of vanilloid receptor located in the brain. Brain Res. Mol. Brain Res. 98:51-57.

57. Mezey, E., et al. 2000. Distribution of mRNA for vanilloid receptor subtype 1 (VR1), and VR1-like immunoreactivity, in the central nervous system of the rat and human. Proc. Natl. Acad. Sci. U. S. A. 97:3655-3660.

58. Winter, J., Dray, A., Wood, J.N., Yeats, J.C., and Bevan, S. 1990. Cellular mechanism of action of resiniferatoxin: a potent sensory neuron excitotoxin. Brain Res. 520:131-140. 